\title{
Maxwell's equations with optical fibers in mind
}

\section{Tevry Freeman}

Tevry Freeman, "Maxwell's equations with optical fibers in mind," Proc. SPIE 2525, 1995 International Conference on Education in Optics, (13 October 1995); doi: 10.1117/12.224071

SPIE Event: SPIE's 1995 International Symposium on Optical Science, Engineering, and Instrumentation, 1995, San Diego, CA, United States 


\title{
Maxwell's Equations with Optical Fibers in Mind.
}

\author{
Terry Freeman \\ School of Mathematics, Physics, Computing and Electronics \\ Macquarie University, New South Wales 2109, \\ AUSTRALIA
}

\begin{abstract}
This study has been conducted in the context of a degree in Optoelectronics, in which a special component on optoelectronics and optical fiber technology, starts in parallel with a conventional second year university physics course. Despite the considerable success of this optics course, it has become clear that there is a need to improve the presentation of topics such as; diffraction and propagation of monochromatic pulses along optical fibers. In this work we seek to structure an outline of the essential principles of light propagation in a non-conducting, non-absorbing dielectric, that is consistent with Maxwell's equations and yet avoids the solutions for conducting waveguides and the other traditional analysis that usually follows. The conditions on the electric field $\mathbf{E}(t)$ and the magnetic field $\mathbf{H}(t)$ applied in the manner prescribed by Diament ${ }^{1}$ leads to a series of field equations for interaction with both perpendicular and oblique plane dielectric surfaces. These equations are then solved numerically to obtain familiar and extended results that illustrate the principles of reflection, refraction and thin-film filters. We aim to introduce the subject of mode dispersion and include a discussion of pulse compression.
\end{abstract}

KEYWORDS: Maxwell's equations, numerical solutions, thin film filters, optical fiber Bragg grating.

\section{INTRODUCTION AND BACKGROUND}

This study is being conducted in the context of a degree in Optoelectronics, in which an initial component of optoelectronics and fiber technology begins in parallel with a conventional second year physics course. While the concepts implicit in Maxwell's equations have been presented from the first year of study, the formal use of these equations is not really expected until students commence their third year. Within the subject of Physics there are many available courses that meet this program, yet for the accelerated Optics programs a real problem exists, because of the nature and timing of the traditional presentations of Maxwell's equations. The teaching of optics at a junior level using Maxwell's equations has been greatly assisted by The Feynman Lectures in Physics ${ }^{2}$, but the thorough pedagogical testing of the alternative viewpoints continues. A more recent model for the presentation of classical optical propagation is found in Wave transmission and Fiber Optics ${ }^{1}$, as the name suggests this book presents a suitable introduction for teaching modern optical fiber principles. 
In the course with which we are concerned, the recommended textbook is Optoelectronics (An introduction) by Wilson and Hawkes ${ }^{3}$, this book gives an broad coverage of the many aspects of the whole year course, but some details require supplementary material. The text covers the topic of Planar dielectric waveguides, which is a useful introduction for propagation along an optical fiber, but in the interests of brevity it starts by quoting Fresnel's equations. The textbook for an earlier parallel physics course, The Physics of Vibrations and Waves by H. J. Pain ${ }^{4}$, has already covered the essential details of the Fresnel equations including "The Impedance of a Dielectric to Electromagnetic Waves". In reviewing the contents and resources of our optoelectronics courses, we decided to search for new paths through this material, that enable us to more quickly reach some of the "state of the art" developments in fiber technology. In particular our interest centered on the properties of optically written fibers studied by a local group (Eggleton et al. $)^{5}$, a topic reviewed by Campbell and Kayshap ${ }^{6}$, or Legoubin et al. ${ }^{7}$. While the search has been successful, we are left with the not unexpected conclusion that elements of computational physics are required to explore the results from the model. This approach is an alternative to the traditional presentation that depends on the available analytic techniques. This report partly addresses the broader question: How can we revise our traditional physics syllabuses by replacing traditional analytical material with computational techniques?

\section{THEORY}

Two of the Maxwell equations can be written in the form

and

$$
\begin{aligned}
& \oint \mathbf{E} . d \mathrm{l}=-\frac{d}{d t} \iint \mathbf{B} . \mathrm{d} \mathbf{S} \\
& \oint \mathbf{H} . d \mathrm{l}=\frac{d}{d t} \iint_{\mathbf{D} . d \mathbf{S}}+\iint \mathrm{J} . d \mathbf{S},
\end{aligned}
$$

we will not be using the magnetic induction vector $\mathbf{B}$, nor the electric displacement vector $\mathbf{D}$. Because we assume propagation in a dielectric that contains no free charge, the electric current density with respect to area, $\mathbf{J}$ is zero. If we also assume that the dielectric is not magnetic we can give the magnetic permeability $\mu$, the same value as that of free space. Next we take a plane wave moving in a $z$-direction, with say the $\mathbf{E}$ vector in the $x$-direction and the $\mathbf{H}$ vector in the $y$-direction. Both these have a factor $\exp (\mathrm{i}(\omega t-n \mathrm{k} z))$, where $\mathrm{k}$ is the wave-number for the radiation in free space, $\omega$ is the angular frequency and $n$ is the refractive index of the medium. By taking suitable rectangular contours for integration with sides along the face of the wave, we obtain

$$
\begin{aligned}
& X\left(E\left(z_{1}\right)-E\left(z_{2}\right)\right)=\frac{\omega \mu}{\mathrm{k} n} X\left(H\left(z_{1}\right)-H\left(z_{2}\right)\right) \\
& Y\left(H\left(z_{1}\right)-H\left(z_{2}\right)\right)=\frac{\omega \varepsilon}{\mathrm{k} n} Y\left(E\left(z_{1}\right)-E\left(z_{2}\right)\right)
\end{aligned}
$$

where $\varepsilon$ is the electric permittivity and $X$ and $Y$ are arbitrary distances along the $x$ - and $y$-directions. With the permeability at the free space value, the first of these equations shows directly that $H \propto n E$, the other gives the relation for radiation resistance $H=\sqrt{\frac{\varepsilon}{\mu}} E$. As both these equations are satisfied we are able to eliminate any, or all integration loops in our isotropic dielectric. We need only apply the equations along dielectric interfaces (where the refractive index changes abruptly) and we need not concern ourselves with the inductive terms found on the right-hand sides of equations (1a) and (1b). The interface equations become 
and

$$
\begin{aligned}
& \oint \mathrm{E} . d \mathrm{l}=0 \\
& \oint n \mathrm{E} . d \mathrm{l}=0
\end{aligned}
$$

where we are able to replace $\mathbf{H}$ with $n \mathbf{E}$ in the nonmagnetic dielectric. Because we are dealing with a plane electromagnetic wave in a non-magnetic medium the Poynting vectors that represent the irradiance are proportional to $n E^{2}$ and are parallel to the propagation axis.

\section{A SINGLE SURFACE}

In terms of our present considerations we can consider the normal incidence of a plane wave on to a flat dielectric surface. The incident and reflected radiation is in a dielectric of refractive index $n_{1}$, while the transmitted radiation is in the dielectric with a refractive index of $n_{2}$. A simple diagram can be used to show the orientation of the electromagnetic fields with respect to the surface and the $z$-axis of propagation.

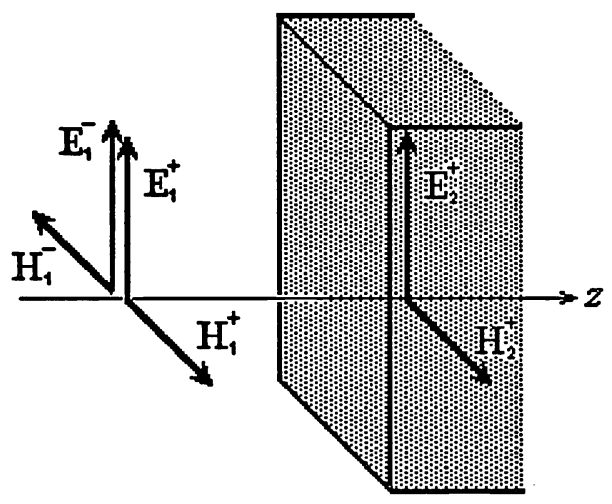

Figure 1

The radiation fields near a dielectric surface.

Now we can apply equations (2a) and (2b) using rectangular contours at the interface, with two sides just outside and inside the surface and the other sides of negligible length penetrating the surface. This procedure gives

and

$$
\begin{aligned}
& E_{1}^{+}+E_{1}^{-}=E_{2}^{+} \\
& n_{1}\left(E_{1}^{+}-E_{1}^{-}\right)=n_{2} E_{2}^{+} .
\end{aligned}
$$

These equations can be extended along with Snell's law to give the Fresnel equations for perpendicular incidence. They also confirm the conservation of the power flux and the reflected wave is found to change phase when reflecting from a medium of greater optical density.

\section{A SINGLE THIN FILM}

We next continue, with the normal incidence of a plane wave on a thin parallel film. The incident, reflected and transmitted radiation is found in an external medium of refractive index $n_{1}$, while the film has a refractive index of $n_{2}$. Figure 2 is modified to show the orientation of the electromagnetic fields with respect to the film and the $z$-axis of propagation. 


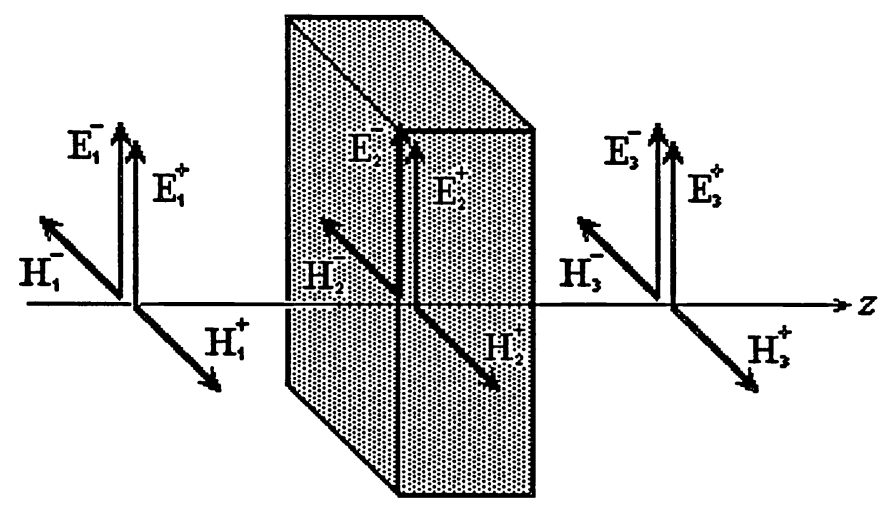

Figure 2

The radiation fields on to and from a dielectric film.

We again apply equations (2a) and (2b) using rectangular contours at each interface to obtain the equations

for one surface and

$$
E_{1}^{+}+E_{1}^{-}=E_{2}^{+}+E_{2}^{-} \text {and } n_{1}\left(E_{1}^{+}-E_{1}^{-}\right)=n_{2}\left(E_{2}^{+}-E_{2}^{-}\right)
$$

$$
E_{2}^{+}+E_{2}^{-}=E_{3}^{+}+E_{3}^{-} \text {and } n_{2}\left(E_{2}^{+}-E_{2}^{-}\right)=n_{1}\left(E_{3}^{+}-E_{3}^{-}\right)
$$

for the other. These equations in themselves do not give sensible results because the unknown radiating fields have arbitrary phase terms; in general they remain complex numbers. If we assume $E_{1}{ }^{+}$is the incident field, we can put $E_{3}^{-}$to zero. $E_{1}^{+}$can be made real and of arbitrary (or unit) amplitude leaving us with four complex equations to describe four complex quantities. While this problem is beyond a simple intuitive approach a physical simplification is available that enables us to proceed; this simplification can be rigorously justified although this is not necessary for this presentation. The simplification is to recognize that each field is generated from the incident field with a common phase at the first surface, except for the field $E_{2}^{-}$, this has reflected back from the second surface. This field is given the appropriate phase correction of $\phi=\mathrm{kn} 22 t$, where $t$ is the thickness of the film.

For the film we can now write

$$
\begin{gathered}
E_{1}^{+}+E_{1}^{-}=E_{2}^{+}+E_{2}^{-} \exp (\mathrm{i} \phi) \\
n_{1}\left(E_{1}^{+}-E_{1}^{-}\right)=n_{2}\left(E_{2}^{+}-E_{2}^{-} \exp (\mathrm{i} \phi)\right) \\
E_{2}^{+}+E_{2}^{-}=E_{3}^{+} \\
n_{2}\left(E_{2}^{+}-E_{2}^{-}\right)=n_{1} E_{3}^{+} .
\end{gathered}
$$

Once more these equations can be easily modified in the appropriate manner for fields that are oblique to the surface. This set of equations can be solved explicitly (although a programmable calculator is advisable) to obtain numerical values for the transimittance and reflectivity of a dielectric film of any thickness, this result is not found in textbooks although Born and Wolf ${ }^{8}$ have a thorough discussion and acknowledge the initial literature for the result.

If the phase shift $\phi$ is set to $\pi$ for a quarter wave film, then we can quickly establish the conservation of energy flow. Perhaps more significantly, we will find that we have a simple solution for the reflected and transmitted fields that automatically includes the contributions from all the internal reflections. 


\section{THE THIN FILM STACK}

For a series of filters of two types, stacked alternately, we can continue the extension of the equations (4). These become

$$
\begin{gathered}
E_{m}^{+}+E_{m}^{-}=E_{m+1}^{+}+E_{m+1}^{-} \exp \left(\mathrm{i} \phi_{2}\right) \\
n_{1}\left(E_{m}^{+}-E_{m}^{-}\right)=n_{2}\left(E_{m+1}^{+}-E_{m+1}^{-} \exp \left(\mathrm{i} \phi_{2}\right)\right)
\end{gathered}
$$

for the $m^{\text {th }}$ interface with the $n_{k} S$ and $\phi_{j} s$ alternating at each step. Some simple algebra puts these equations into a suitable form to derive the characteristic matrices for each interface (assuming normal incidence):

$$
\begin{aligned}
& E_{m}^{+}=\frac{1}{2}\left(1+\frac{n_{2}}{n_{1}}\right) E_{m+1}^{+}+\frac{1}{2}\left(1-\frac{n_{2}}{n_{1}}\right) \exp \left(\mathrm{i} \phi_{2}\right) E_{m+1}^{-} \\
& E_{m}^{-}=\frac{1}{2}\left(1-\frac{n_{2}}{n_{1}}\right) E_{m+1}^{+}+\frac{1}{2}\left(1+\frac{n_{2}}{n_{1}}\right) \exp \left(\mathrm{i} \phi_{2}\right) E_{m+1}^{-}
\end{aligned}
$$

It is about this point that most textbooks start to end the discussion by looking at particular solutions with $\phi=n \pi$ ( $n$ being an integer). With the modern computational aids available to students it is now possible to continue the discussion. For instance, even the student edition of MatLab is designed to handle small matrices with ease. Simpler still, the calculations can be continued with complex algebra on moderate sized programmable calculators. As an example the programs for this paper have been written and tested on a Texas Instrument's T. I. 74, mostly while access to other computers was inconvenient.

The initial conditions are important for the solution, the conventional approach is to set the incident field $E_{1}^{+}$to $E_{0}$ (which is real and of convenient amplitude), unfortunately the other initial field $E_{1}^{-}$is both imaginary and of unknown amplitude. At the other end of the stack the transmitted field, say $E_{\mathrm{M}}{ }^{+}$is also imaginary and of unknown amplitude. For our purposes we initially assume that $E_{\mathrm{M}}{ }^{+}=1$ and work backwards through the filter stack, when we have reached $E_{1}^{+}$and $E_{1}^{-}$we renormalise our values of $E_{\mathrm{M}}{ }^{+}$ (originally 1 ), $E_{1}^{+}$and $E_{1}^{-}$so that $E_{1}^{+}=1$. From the new values of $E_{\mathrm{M}}^{+}$and $E_{1}^{-}$we can calculate the reflectance and transmittance of the stack. To test the solution these should total one. In cases where the transmittance approaches zero, the calculation exceeds the ability of numerical precision of a typical calculator or program language. There are ways of overcoming this problem, but these are not part of this illustrative work. As a general rule the calculations proceed quite well for about one hundred dielectric films of refractive index 1.5.

The program is easily tested by finding the otherwise known results for a single thin film. Once this test is passed, it is instructive to systematically add a film at a time to the stack and discuss the results. Rather than consider individual results a plotting routine was added, this takes alternate films of $n_{1}=1.0$ and $n_{2}=1.5$ in a surrounding medium of $n_{1}$. It starts plotting with the all films of zero thickness and expands them uniformly, so that they have a common optical thickness $\phi_{1}=\mathrm{k}_{1} 2 t_{1}=\phi_{2}=\mathrm{k} n_{2} 2 t_{2}$. The results of this calculation are shown in figure 3 , where the stack has been started with one film and increased to nine. 


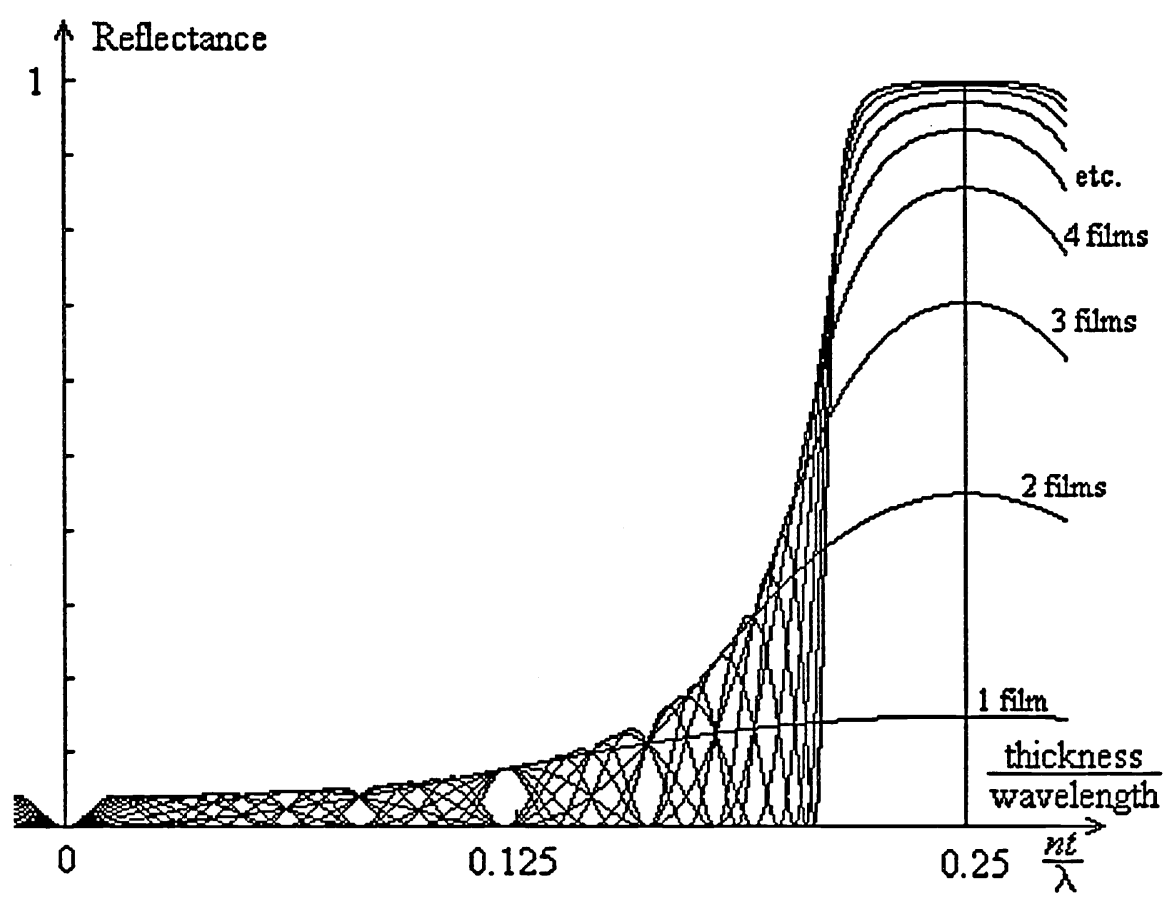

Figure 3.

The reflectance of one to nine thin films of refractive index 1.5 separated in air and drawn out so that the optical thickness divided by wavelength varies.

\section{A MODULATED INDEX DIELECTRIC}

Having set up the intitial computer program, the next step was to modify it so that we can use it to discuss some of the modern developments in fiber technology. The example selected was to attempt to model a chirped optical fiber Bragg grating ${ }^{5}$. These gratings are optical fibers in which the refractive index varies sinusiodally with a small amplitude along the propagation axis. The filter stack was a first approximation to this system. The next program can be easily modified to produce any desired longitudinal profile of refractive index, however we started with the sinusoidal variation because it could be directly compared with the previous square wave model.

The new program uses the equations

and

$$
\begin{aligned}
& E_{m}^{+}=\frac{1}{2}\left(1+\frac{n_{m+1}}{n_{m}}\right) E_{m+1}^{+}+\frac{1}{2}\left(1-\frac{n_{m+1}}{n_{m}}\right) \exp \left(\mathrm{ik} n_{m} 2 t\right) E_{m+1}^{-} \\
& E_{m}^{-}=\frac{1}{2}\left(1-\frac{n_{m+1}}{n_{m}}\right) E_{m+1}^{+}+\frac{1}{2}\left(1+\frac{n_{m+1}}{n_{m}}\right) \exp \left(\mathrm{ik} n_{m} 2 t\right) E_{m+1}^{-}
\end{aligned}
$$


For each iteration, a new value of the refractive index $n_{m}$ is calculated from a pre-programmed function and the computer works backwards as before. This time as we are integrating, it is neccessary to use very small steps, at least ten per cycle of the refractive index. As a consequence the full plotting routine is too slow for class use. It is however be useful for private investigation. In cases where the final transmittance approaches zero we again have the numerical problem when values of $E_{m}$ overload.

To test this program, we have calculated first the reflectance from a stack of thirty thin films of refractive index 1.5 with air spacing, and then we have found the reflectance for a ray, that encounters a dielectric that has an mean refractive index of 1.25 and a sinusoidal variation of amplitude of 0.25 , along the propagation axis of the normal wave or ray.

The result for the thirty film filter stack is shown (with similar graphic axes to those used in figure 3) in figure 4. As the optical thickness of each film divided by the wavelength runs past 0.25 the pattern of the reflectance repeats periodically, until the whole system becomes metres thick. In figure 5 we have shown the matching case where a dielectric varies sinusoidally for thirty cycles. Although the results are virtually identical, the reflectance does not repeat periodically beyond an optical thickness of one quarter of a wavelength.

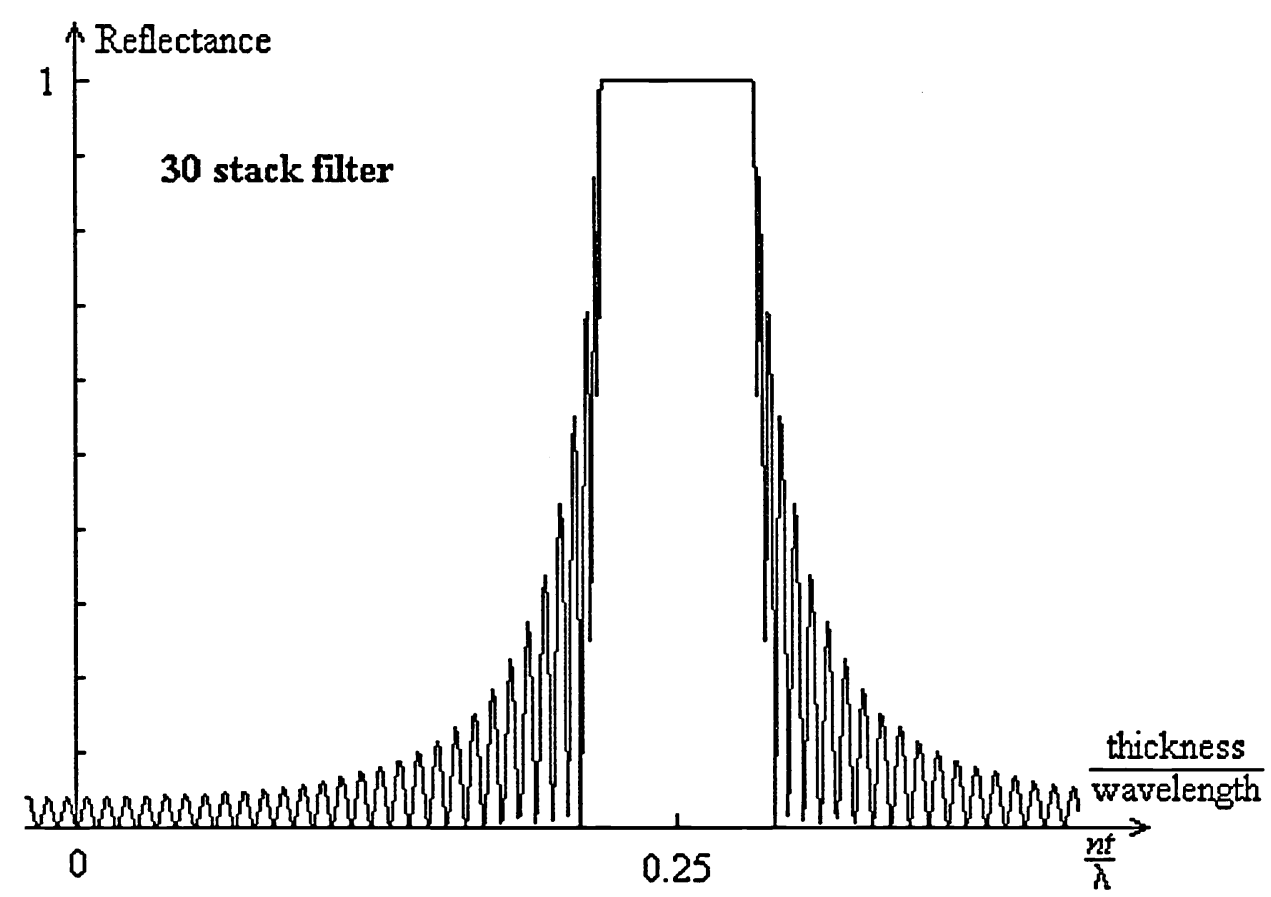

Figure 4.

The reflectance from 30 thin films of refractive index 1.5 in air as the stack is uniformly increased in optical thickness. 


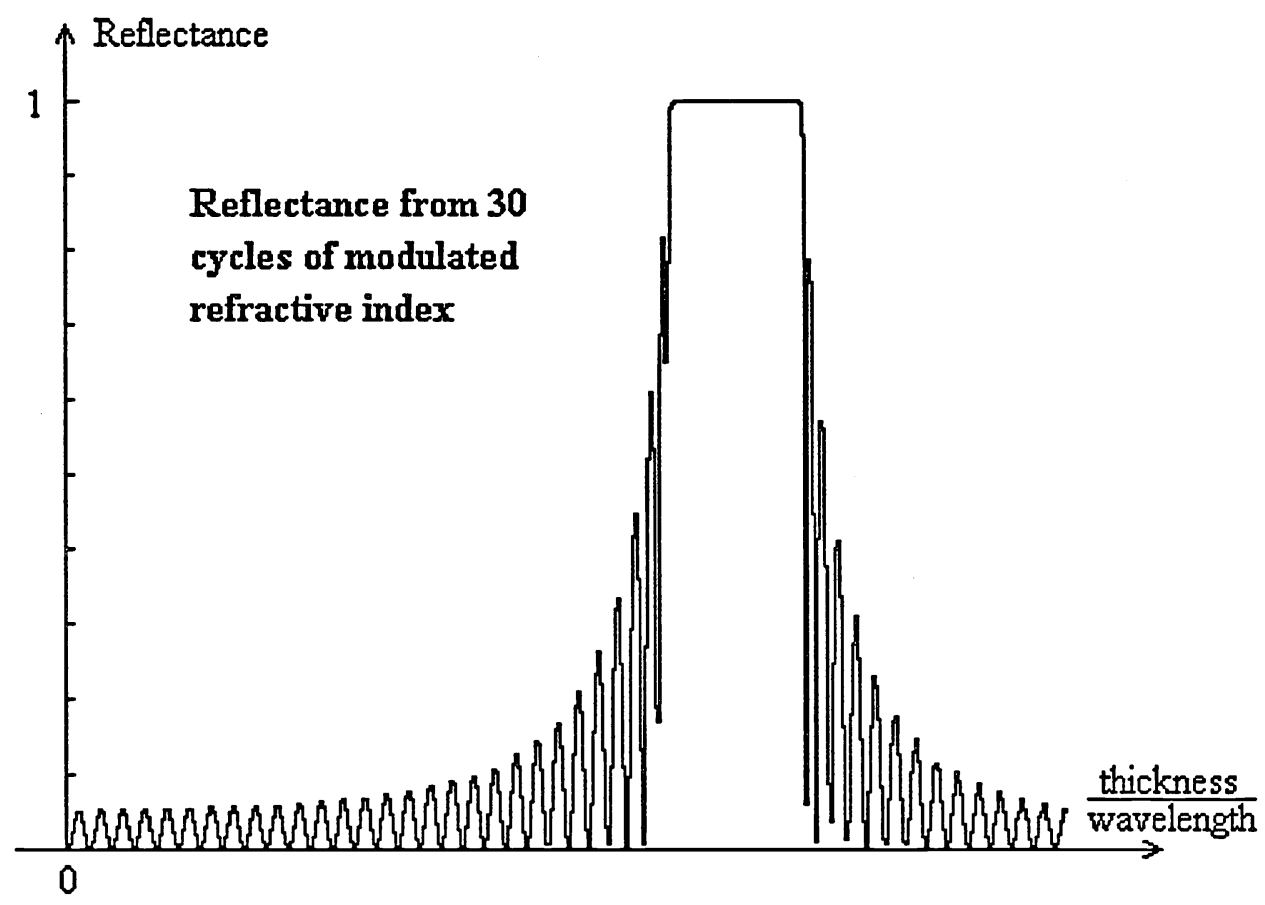

Figure 5.

The reflectance from a dielectric with a modulated refractive index of $n=1.25+0.25 \sin (\mathrm{kz})$.

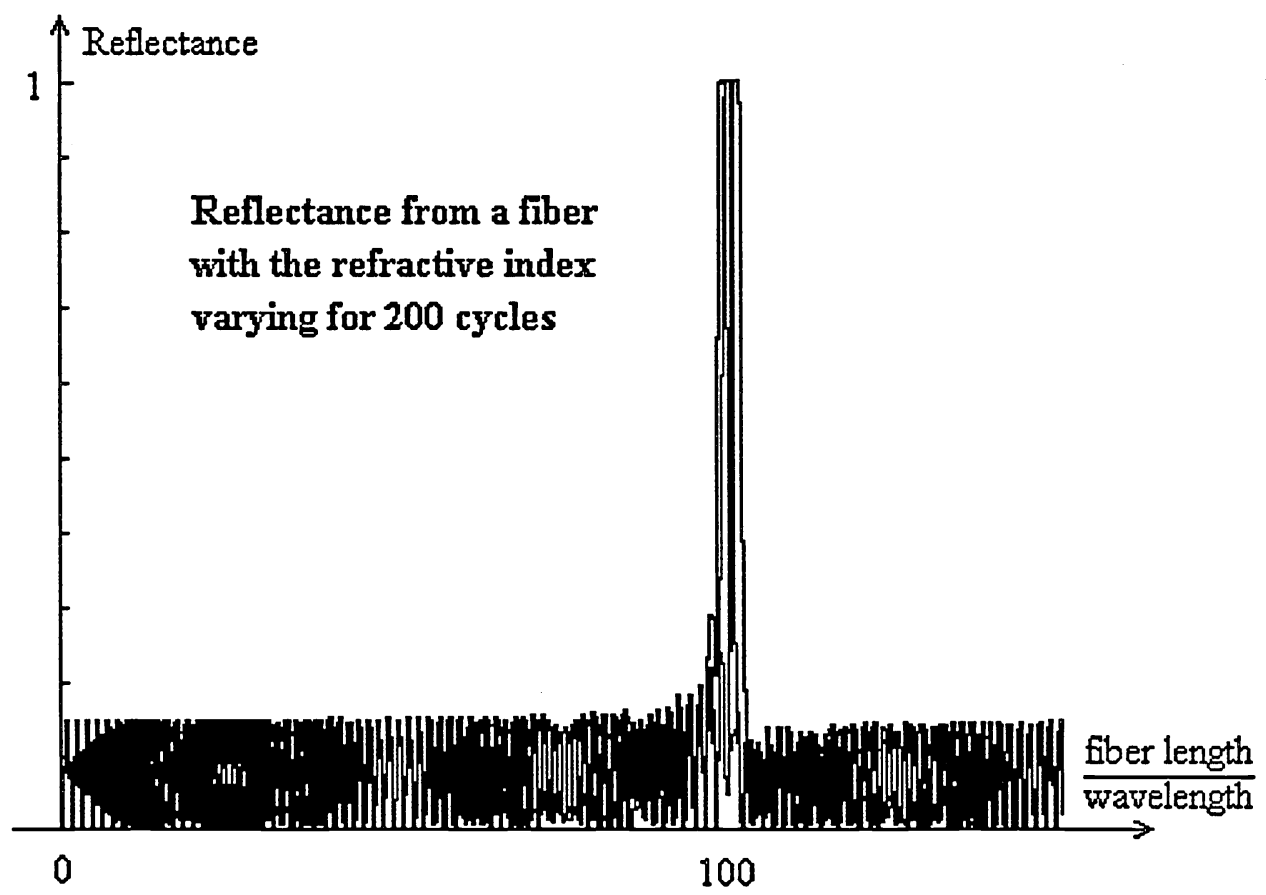

Figure 6.

The reflectance from a dielectric with a modulated refractive index of $n=1.50+0.02 \sin (\mathrm{kz})$. 
In figure 5 it is not a simple matter to scale the optical length of a system with a variable refractive index, the diagrams use reciprocal wavelength scales that give similar figures. Finally the program was used to reconstruct an optical fiber Bragg grating. The average refractive index was 1.50 and this was varied with an amplitude of 0.02 for 200 cycles, the fibre was then drawn out on a scaled length as before. The result is shown in figure 6 . In this figure there was a problem with the fine structure interfering against the background bitmap, to reduce this effect three nearly equivalent structures have been superimposed.

\section{CONCLUSIONS}

The original starting point from equations (1a) and (1b) have proved to be successful, the approach is not original, but is not commonly found in text books. Most of the preliminary assumptions are no more formidable than those needed for other more conventional approaches to Maxwell's equations. From the early stages of the course it is comparatively easy to demonstrate the half wavelength phase change on reflection from a surface of greater refractive index. The multiple pass, internal reflection model, of division of amplitude interference, is replaced by a simple model that can be extended to give other results that presently tend to be given as axiomatic. By the time the repertoire is starting to become depleted, the computational model can be introduced; this is particularly suitable for undergraduates using student versions of mathematical packages. The path is especially appropriate for students of optical sciences and engineering because it leads towards the subjects of optical interference and the properties of reflection that are necessary for present high technology applications.

As the course is being slowly adapted in the context of present syllabuses there is no need for a speedy introduction. The instructor needs to be aware of the student's difficulties and fears and these are already becoming apparent. The first problem encountered is the perception of the students that computing is for computing studies, while difficult mathematical analysis is the lot of the physics student. We have encountered examples where able students are unwilling to use their computing skills because they would rather enhance their marks with traditional assessment tasks. The second major problem is the conservatism of students, if they can't find the theoretical outline in a textbook then the course is to be viewed with suspicion. This is understandable as students are approaching the subject for the first time and they have little appreciation of the difficult areas and stumbling blocks that have been encountered in the past. For this reason the course is evolving slowly and we trust surely, the fresh approach seems to sit nicely in the evolving syllabus.

\section{ACKNOWLEDGMENTS}

This study and the preparation of course materials have been fully supported by a Macquarie University Teaching Research Grant. 


\section{REFERENCES}

1. P. Diament, Wave Transmission and Fiber Optics, Macmillan Publishing company, New York, 1990.

2. R. P. Feynman, R. B. Lieghton and M. Sands, The Feynman Lectures in Physics, volumes I and II, Addison-Wesley Publishing Co., Reading Massachusetts, 1963.

3. J. Wilson and J. F. B. Hawkes, Optoelectronics An Introduction, Prentice Hall, London, 2nd edition 1989.

4. H. J. Pain, The Physics of Vibrations and Waves fourth ed., Wiley, Chichester U. K. 1993.

5. B. J. Eggelton, P. A. Krugg, L. Poladian, K. A. Ahmed and H. F. Liu, "Experimental demonstration of compression of dispersed optical pulses by reflection from self-chirped optical fiber Bragg gratings", Optics Letters, vol. 19, no. 12, pp. 877-879, 1994.

6. R. J. Campbell and R. Kayshap, "The properties and applications of photosensitive germanosilicate fibre”, Int. Journal of Optoelectronics, vol. 9, no. 1, pp. 33-57, 1994.

7. S. Legoubin, M. Douay, P. Bernage, P. Niay, J. F. Bayon, T. Georges, "Photoinscription de filtres de Bragg dans des fibres optiques dopees a l'oxyde de germanium”, Journal Optics (Paris), vol. 23, no. 4, pp 143-156, 1992.

8. M Born and E. Wolf, Principles of Optics, Electromagnetic Theory of Propagation Interference and Diffraction of Light, Sixth edition, Maxwell Macmillan, Pergamon Press, Oxford, 1985. 